\title{
How can I tackle microaggressions in the workplace?
}

It can be hard to know how to tackle these subtle but damaging behaviours, but there are ways to do it, experts tell Abi Rimmer

\section{Abi Rimmer}

The BMJ

\section{"It takes courage"}

Sue Crossland, consultant in acute medicine and president of the Society for Acute Medicine, says, "Recent discussions on Twitter and with my colleagues suggest that microaggressions are a significant risk factor for diminishing wellbeing and productivity at work. Microaggressions are not solely directed at women, but it's on this perspective, my own, that I am going to focus.

"Microaggression can be defined as a statement, action, or incident of indirect, subtle, or unintentional discrimination against a marginalised group. Often, in isolation they appear harmless, and critics have suggested that they are trivial and negligible, likening them to everyday incivility or rudeness. It is, however, the day to day constancy of microaggressive assaults that make them gradually erode the wellbeing and resilience of the victim.

"As a senior consultant and the president of a specialist society, my expertise in my chosen field should be clear. And yet, daily, I am faced with subtle microaggressions designed to make me think that my decisions are flawed; that I am emotional and hysterical; or that my input is not valued. I recall a crowded meeting with mostly male colleagues. While they didn't all agree with each other, each was given an opportunity to speak. As I began to offer my opinion, the chair of the meeting rolled his eyes and said, 'Here we go again.' As I started to formulate a response, he immediately interrupted and began talking over me. How did I feel? Belittled and like an impostor. Afraid to speak out.

"How do we deal with this? It's not good enough to sigh inwardly. It's not good enough to be silenced. But it takes courage to challenge these behaviours-especially, as is already noted above, to other members of the team they can seem isolated and trivial.

"I calmly waited until he had finished speaking and said, "May I continue to contribute?' There was embarrassed silence, followed by some blustering. There may have been some 'mansplaining,' which is synonymous with microaggression against women. I never did get my point across, but I hope that some of my colleagues were able to reflect on this uncomfortable encounter and make a decision to behave in a more constructive way. I live in hope and optimism.”

\section{"Weigh up the risks and benefits of tackling microaggressions"}

Oluseyi Adesalu, medical education fellow at Health Education England and honorary research assistant at research department for medical education at UCL, says, "As a junior doctor it can be easy to forget that it's your right to work in an environment that is free from discrimination or marginalisation. Unlike the guidelines available to us when managing a deteriorating patient, tackling microaggressions is something we are forced to learn implicitly, through our interactions with patients and colleagues.

"In truth, as a junior doctor, challenging microaggressions is an art I am yet to master. The variety and nuance that underpin the nature of microaggressions makes identifying and educating others about them particularly difficult. Whether it be from a patient inquiring if you are intending to go 'back to where you came from' after training or a consultant attempting a joke about 'you people,' prioritising your immediate professional and personal wellbeing is important. With this in mind, my approach currently involves weighing up the risks and benefits of tackling each microaggression as it comes.

"The way this looks will be different for everyone. Examples include asking the person at the time to stop, turning the question or statement back on them, or discussing it with someone you trust after it has happened. All of these approaches can be challenging and are not without pitfalls, such as the risk of being dismissed as too sensitive or confrontational.

"Unfortunately, the burden of tackling microaggressions in the workplace falls on those they are directed against. It's everyone's responsibility, however, to create a workplace culture that is both inclusive and nurturing to all. This occurs through careful consideration of our words, silences, actions, and inactions. Therefore, we all have a role in reflecting on how we can use the privileges we have to educate ourselves on challenging microaggressions and removing them from the workplace altogether."

\section{"Encourage dialogue rather than recriminations"}

Val Yeung, consultant child and adolescent psychiatrist, says, "Rather than reacting immediately, discuss the incident with a trusted colleague. This can help you to validate and harness 
your emotions, allowing you to constructively respond to the incident. Approaching the situation from a position of curiosity, from which all involved can learn and change, can take the heat out of discussions.

"Consider your role in the incident, and also in the team and organisation within which it occurred. Were you directly affected, or a witness? Bearing these things in mind, consider what message you would like conveyed to the instigators, and the best person to do this. Should this be brought up in person or by email? Remember that your own beliefs and biases could help or hinder things.

"Before confronting the instigators, consider how you will have the conversation, and protect the space and time for this. Do other people need to be present? Be mindful of the language you use, its tone, and content. Invite open dialogue to minimise defensiveness or feelings of being accused by focusing on the incident in hand; the comments and behaviours and how they made you feel. Ask things like, 'Do you have any ideas why that was upsetting?' or 'What would be helpful for us to do to stop this from happening again?'

"I've previously used approaches from family therapy: externalising, circular questioning, and coordinated management of meaning. These help to use empathy to understand different perspectives at multiple levels, clarify the differences between impact and intent relationally, and reinforce ongoing collaborative teamwork within an inclusive culture of learning for all.

"Also consider possible wider approaches to repeated microaggressions by reviewing your Dignity and Respect at Work policy, and any need for timely human resources advice."

\section{"Don't apologise for who you are"}

Shareen Pavaday, head of equality, diversity, and inclusion at North Middlesex University Hospital NHS Trust, says, "Have you ever been in a situation where you've been complimented on 'speaking English well,' even though it's your first language? Have you ever been called 'nurse' even though you're the doctor? Have you ever been asked to get the senior professional's attention when he's actually the student radiographer? What do you do?

"Having spent a lifetime convincing myself that my skin colour doesn't matter because I was born and raised in Britain and therefore I should have the same opportunities as anyone else, I now find myself in a role where I'm learning that has never been true.

"To then be faced with what I now understand to be microaggressions, I can see that people's ignorance is no longer a reason to excuse the apathy that comes with it.

"I welcome people's curiosity about my story because I am extremely proud of it. But if you are really interested in what I am and where I come from you will ask the right questions. You will also accept the answers and not roll your eyes with a follow on of, 'No, you know what I mean.' No, I don't.

"Self-identity can be a tough one to crack, so if you are finding yourself surrounded by these questions make sure you know who you are before trying to correct or educate others. Own your space and be unapologetic about yourself-only then will you be satisfied with your answers even if the other person isn't. "Fundamentally, don't allow someone else to define who or what you are. To use an African philosophy that now resonates with me-Ubuntu: I am because we are.”

\section{'Patients' behaviour is often based in fear"}

Indranil Chakravorty, consultant physician at St George's University of London, says, "Healthcare is an emotive business that brings out the very best and worst in people. Expectations often don't match what is available and in the chaos that ensues, trust and respect are often lost.

"Healthcare workers are trained to be objective, professional, ethical, and empathetic during such interactions. However, the stark reality of providing healthcare means that mutual respect and professionalism often break down.

"What, then, are the strategies for managing to control such situations, while maintaining objectivity and composure? The first rule is to assume that the aggrieved patient is here because of a desperate need for care and is most likely anxious, distressed, and afraid. The stance we need to take as doctors, therefore, is one of calm, composed reassurance.

"Active listening is most valuable. I tend to let my patients or their relatives pour their hearts out while I listen to everything they have to say. I try to offer a solution that fits their agenda, irrespective of whether I think, clinically speaking, it is relevant to the presentation. Most patients have a fairly good idea of what they think is wrong with them and often are determined to get what they think is the best treatment for their condition.

"A patient with a cough, for example, might ask for an antibiotic course from their GP or come to my practice wanting a computed tomography scan. If one were to dismiss such requests, the common conclusion would be that the doctor or nurse was not taking the condition seriously. If you asked the patient what they were most worried about, however, the answer might relate back, for example, to cancer that was discovered too late in a close relative or acquaintance.

"There is always an underlying reason fuelling microaggressive responses. Once these are heard and responded to with reassurance, usually only for a few minutes, the scene is set for a more clinically oriented discussion and I find my patients are much more receptive.

"Finally, in spite of all the measures described here, there will always be some people who thrive on aggression and violence. You need to know when the risks are too high, remember the basics of self-preservation, and withdraw to a safe distance, but without any reciprocal aggression.

"Ultimately, we are here for a reason. Our mission and vocation is to do the best we can for our fellow humans. We all have to work with people who are angry or aggressive, but I believe these behaviours are manifestations of an inner fear of loss or the unknown. We have a duty to offer our deepest empathy and reassurance." 